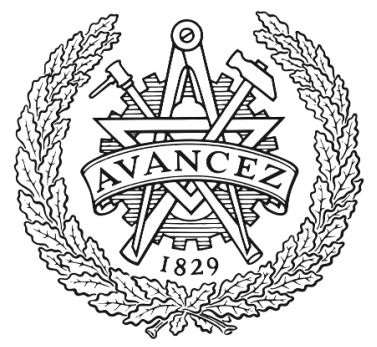

CHALMERS

UNIVERSITY OF TECHNOLOGY

\title{
Synthesis and Electronic Properties of Diketopyrrolopyrrole-Based Polymers with and without Ring-Fusion
}

Downloaded from: https://research.chalmers.se, 2023-04-26 14:13 UTC

Citation for the original published paper (version of record):

Zhuang, W., Wang, S., Tao, Q. et al (2021). Synthesis and Electronic Properties of Diketopyrrolopyrrole-Based Polymers with and without

Ring-Fusion. Macromolecules, 54(2): 970-980. http://dx.doi.org/10.1021/acs.macromol.0c02326

N.B. When citing this work, cite the original published paper. 


\title{
Synthesis and Electronic Properties of Diketopyrrolopyrrole-Based Polymers with and without Ring-Fusion
}

\author{
Wenliu Zhuang, ${ }^{\ddagger}$ Suhao Wang, ${ }^{\ddagger}$ Qiang Tao, Wei Ma, Magnus Berggren, Simone Fabiano,* \\ Weiguo Zhu,* and Ergang Wang*
}

Cite This: Macromolecules 2021, 54, 970-980

Read Online

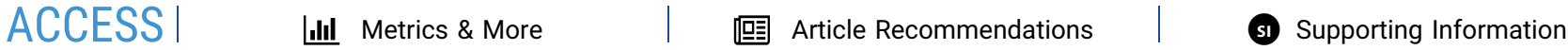

ABSTRACT: Diketopyrrolopyrroles (DPP) have been recognized as a promising acceptor unit for construction of semiconducting donor-acceptor $(\mathrm{D}-\mathrm{A})$ polymers, which are typically flanked by spacers such as thiophene rings via a carbon-carbon single bond formation. It may suffer from a decrease in the coplanarity of the molecules especially when bulky side chains are installed. In this work, the two $\mathrm{N}$ atoms in the DPP unit are further fused with C-3 of the two flanking thiophene rings, yielding a $\pi$-expanded, very planar fused-ring building block (DPPFu). A novel DPPFu-based D-A copolymer (PBDTT-DPPFu) was successfully synthesized,

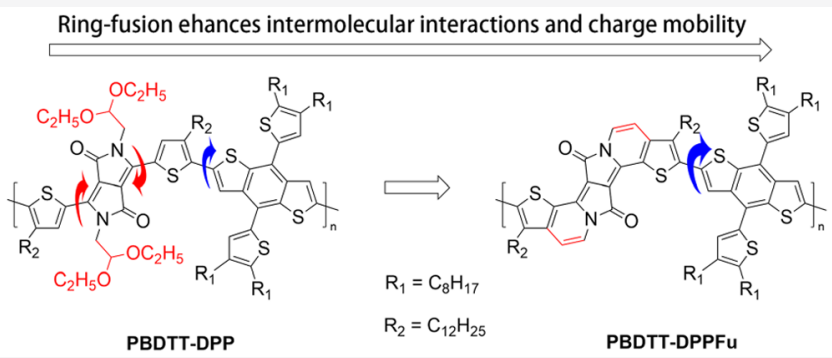
consisting of a benzo $\left[1,2-b: 4,5-b^{\prime}\right]$ dithiophene (BDTT) unit as a donor and a DPPFu unit as an acceptor. For comparison, the unfused DPP-based counterpart PBDTT-DPP was also synthesized. Two dodecyl alkyl chains were attached to thiophene rings of DPP moieties to ensure good solubility of the DPPFu-based polymer. The influence of the ring-fusion effect on their structure, photophysical properties, electronic properties, molecular packing, and charge transport properties is investigated. Ring-fusion enhances the intermolecular interactions of PBDTT-DPPFu polymer chains as indicated by density functional theory calculation and analysis of electrostatic potential and van der Waals potential and results in significantly improved molecular packing for both the inplane and out-of-plane directions as suggested by X-ray measurements. Finally, we correlate the molecular packing to the device performance by fabricating field-effect transistors based on these two polymers. The charge carrier mobility of the ring-fused polymer PBDTT-DPPFu is significantly higher as compared to the PBDTT-DPP polymer without ring-fusion, although PBDTT-DPPFu exhibited a much lower number-average molecular weight of $17 \mathrm{kDa}$ as compared to PBDTT-DPP with a molecular weight of 108 $\mathrm{kDa}$. The results from our comparative study provide a robust way to increase the interchain interaction by ring-fusion-promoted coplanarity.

\section{INTRODUCTION}

Solution-processable semiconducting polymers have attracted wide attention thanks to their versatile chemical synthesis and the opportunity for low-cost fabrication of large-area flexible devices such as smart cards, bendable displays, radiofrequency identification (RFID) tags, and distributed sensors. ${ }^{1,2}$ In recent years, great efforts in molecular design have boosted the critical field-effect charge mobility of the conjugated polymers, with values now exceeding $10 \mathrm{~cm}^{2} \mathrm{~V}^{-1} \mathrm{~s}^{-1}$. ${ }^{3-9}$ One of the successful keys to achieve high mobility in semiconducting polymers is to maximize the intra- and intermolecular charge transport by means of improving: (i) polymer backbone coplanarity, ${ }^{3,10}$ (ii) molecular weight, ${ }^{1,12}$ (iii) orientation of polymer chains, ${ }^{13-22}$ and (iv) intermolecular interactions between neighboring molecules. ${ }^{23}$ In line with such considerations, donor-acceptor (D-A) copolymers have shown great potential owing to their tunable energy levels, strong intramolecular charge transfer (ICT), and intermolecular interactions between adjacent polymer chains. ${ }^{24-27}$ Moreover, recent experimental evidences have shown that local aggregation in this class of polymers over just a few chains is a sufficient mesoscopic structure to ensure high mobility and thus desired swift operation in organic fieldeffect transistors (OFETs). ${ }^{28}$

Diketopyrrolopyrroles (DPP) have been recognized as a promising acceptor unit for high-performance semiconducting polymers due to their tight $\pi-\pi$ stacking and long-range order that is induced by the high coplanarity and cross-axis dipole. ${ }^{8,29,30}$ In the past decade, the incorporation of DPP into polymers for OFETs has attracted much attention in academe, with charge carrier mobility surpassing $10 \mathrm{~cm}^{2} \mathrm{~V}^{-1}$ $\mathrm{s}^{-1}$. $7,8,31-33$ They are typically flanked by spacers such as thiophene rings via a carbon-carbon single bond formation. ${ }^{8,9,34-37}$ However, when bulky side chains are needed and

Received: October 13, 2020

Revised: December 25, 2020

Published: January 11, 2021

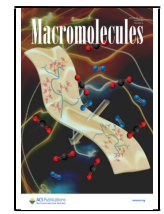


Scheme 1. Synthetic Routes to the Polymers PBDTT-DPP and PBDTT-DPPFu

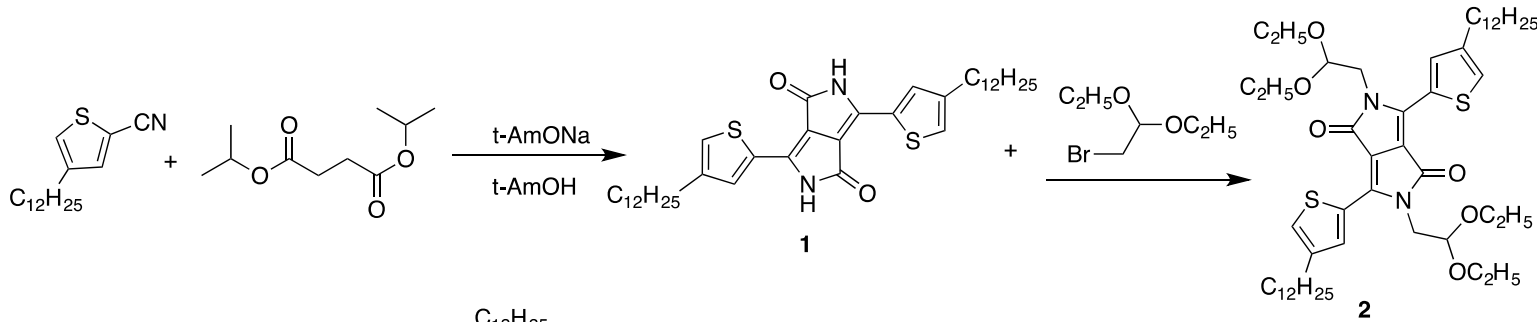

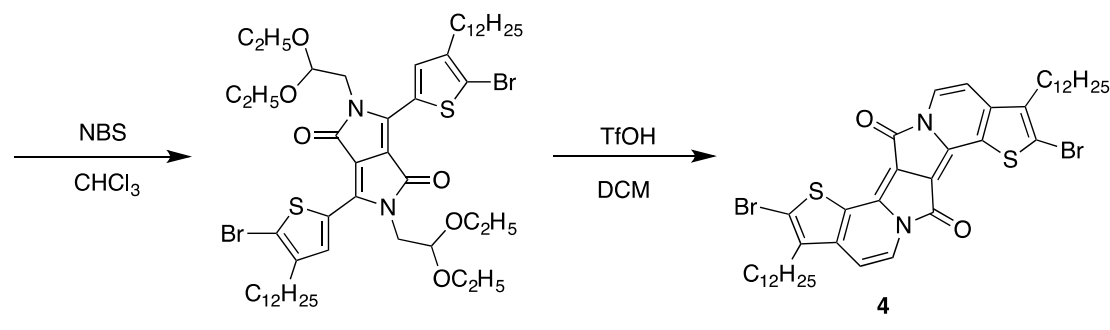

3

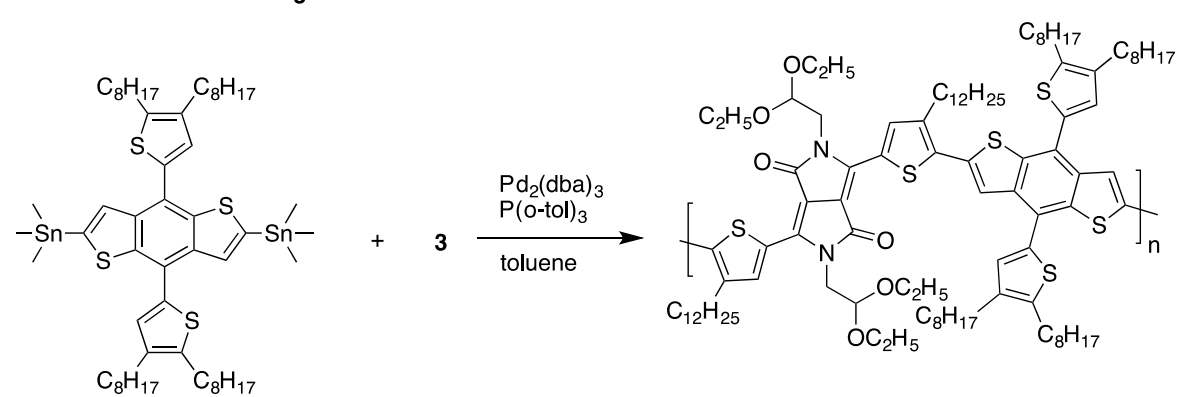

PBDTT-DPP

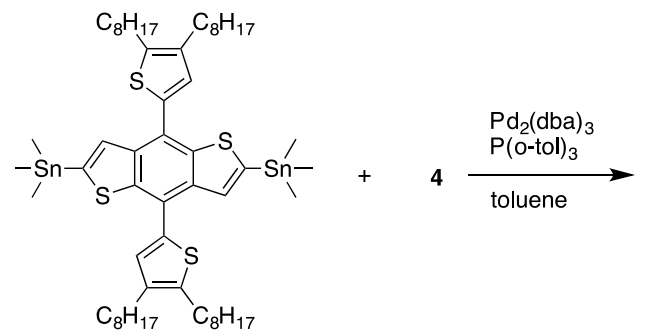

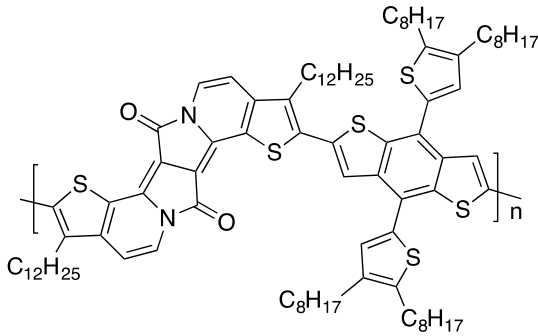

PBDTT-DPPFu installed to prepare soluble conjugated polymers, they may suffer from a decrease in the coplanarity of the molecules. Making the backbone rigid and planar by means of ring-fusion can be an effective method under such occasions. Gryko and co-workers reported a synthetic approach for S-shaped $\pi$ expanded DPP analogues with very high overall yield. ${ }^{38}$ Such a method facilitates the introduction of electron-rich, or electron-neutral, aryl or heteroaryl rings within the DPP scaffold. A strong bathochromic shift of the absorption maxima was observed as a result of the extended conjugation length. ${ }^{38}$ Chen et al. also reported a novel thiophene-fused DPP unit (PTI) to construct D-A small molecules for organic photovoltaics (OPV). ${ }^{30}$ Shi et al. reported a half-fused DPPbased $\mathrm{D}-\mathrm{A}$ polymer showing redshifted absorption. ${ }^{39}$ Until recently, Scherf et al. reported a series of DPPFu-based D-A polymers with promising absorption and emission properties. ${ }^{40}$ So far, research efforts are still needed to further explore the potential of ring-fused DPP-based polymers. To this purpose, ring-fused DPP-based polymers are expected to induce a strong intermolecular interaction due to extended conjugation along the backbone and retainment of high molecular coplanarity as compared to conventional DPP-based copolymers with bulky side chains. ${ }^{39,40}$

Herein, we designed and synthesized two new DPP-based $\mathrm{D}-\mathrm{A}$ copolymers for a comparative study. The two $\mathrm{N}$ atoms in the DPP unit are fused with C-3 of the two flanking thiophene rings, yielding a $\pi$-expanded, planar fused-ring building block (DPPFu). A novel DPPFu-based D-A copolymer (PBDTTDPPFu) was successfully synthesized, consisting of a benzo$\left[1,2-b: 4,5-b^{\prime}\right]$ dithiophene (BDTT) unit as a donor and a $\mathrm{DPPFu}$ unit as an acceptor. For comparison, the unfused DPPbased counterpart PBDTT-DPP was also synthesized (Scheme 1). Two dodecyl alkyl chains were attached to thiophene rings of DPP moieties to ensure solubility of the resulting polymers for high molecular weights. The influence of ring-fusion on their structure, photophysical properties, molecular packing, and charge transport properties is investigated. Ring-fusion increases the planarity of PBDTT-DPPFu polymer chains and results in significantly improved molecular packing for both inplane and out-of-plane directions. Finally, the molecular packing is correlated to the device performance by fabricating OFETs based on these two polymers. The charge carrier 
Table 1. Molecular Weights and Optical and Electrochemical Properties of the Polymers

\begin{tabular}{|c|c|c|c|c|c|c|c|c|}
\hline \multirow[b]{2}{*}{ polymer } & \multirow[b]{2}{*}{$M_{\mathrm{n}}(\mathrm{kDa})$} & \multirow[b]{2}{*}{$\bigoplus_{M}$} & \multirow{2}{*}{$\frac{\text { solution }}{\lambda_{\text {abs }}(\mathrm{nm})}$} & \multicolumn{2}{|c|}{ film } & \multirow[b]{2}{*}{ HOMO $(\mathrm{eV})$} & \multirow[b]{2}{*}{ LUMO $(\mathrm{eV})$} & \multirow[b]{2}{*}{$E_{\mathrm{g}}^{\mathrm{ec}}(\mathrm{eV})^{l}$} \\
\hline & & & & $\lambda_{\text {abs }}(\mathrm{nm})$ & $E_{\mathrm{g}}^{\text {opt }}(\mathrm{eV})^{a}$ & & & \\
\hline PBDTT-DPP & 108 & 2.3 & 660 & 764 & 1.52 & -5.66 & -3.97 & 1.69 \\
\hline PBDTT-DPPFu & 17 & 2.0 & 710 & 758 & 1.49 & -5.50 & -3.88 & 1.62 \\
\hline
\end{tabular}

${ }^{a}$ Optical gap estimated from the optical absorption edge of the film. ${ }^{b} E_{\mathrm{g}}^{\mathrm{ec}}=$ LUMO - HOMO.

mobility of the ring-fused polymer PBDTT-DPPFu is significantly improved as compared to the PBDTT-DPP polymer without ring-fusion.

\section{RESULTS AND DISCUSSION}

Material Synthesis. The synthetic routes of the polymers PBDTT-DPP and PBDTT-DPPFu are shown in Scheme 1. The preparation of monomers $1-4$ is described in the Supporting Information. The polymerization was realized via $\mathrm{Pd}_{2}(\mathrm{dba})_{3}$-catalyzed Stille coupling of bis(trimethylstannyl)benzo[1,2- $\left.b: 4,5-b^{\prime}\right]$ dithiophene with dibromo-substituted monomers 3 or 4 , respectively. The reaction mixture was refluxed in toluene with vigorous stirring for $24 \mathrm{~h}$. Further purification was carried out by Soxhlet extraction and column chromatography. Both polymers are readily soluble in organic solvents such as chloroform, toluene, and o-dichlorobenzene (oDCB) thanks to the long dodecyl chains of the DPP moieties. The choice of dodecyl chains is a trade-off between polymer solubility for high molecular weights and backbone planarity for favorable interchain interactions. We also synthesized a DPPFu monomer without any side chains but did not succeed in obtaining a soluble polymer out of it with high enough molecular weights. The molecular weights of the polymers were determined by size exclusion chromatography (SEC). The molecular weights $\left(M_{\mathrm{n}}\right)$ and molar mass dispersity $\left(\oplus_{M}\right)$ are listed in Table 1 . The ring-fused monomer 4 exhibits decreased solubility, which results in a much lower $M_{n}$ of the polymer PBDTT-DPPFu $(17 \mathrm{kDa})$ as compared to that of PBDTT-DPP (108 kDa). Both polymers exhibit a small $\bigoplus_{M}$ (2.0 for PBDTT-DPPFu and 2.3 for PBDTT-DPP). A doubled number of alkyl side chains may have to be attached in order to obtain PBDTT-DPPFu with molecular weight as high as PBDTT-DPP.

Optical and Electrochemical Properties. The normalized UV-vis absorption spectra of PBDTT-DPP and PBDTT$\mathrm{DPPFu}$ in chloroform solution and in film are shown in Figure 1. Both polymers exhibit two distinct high and low energy

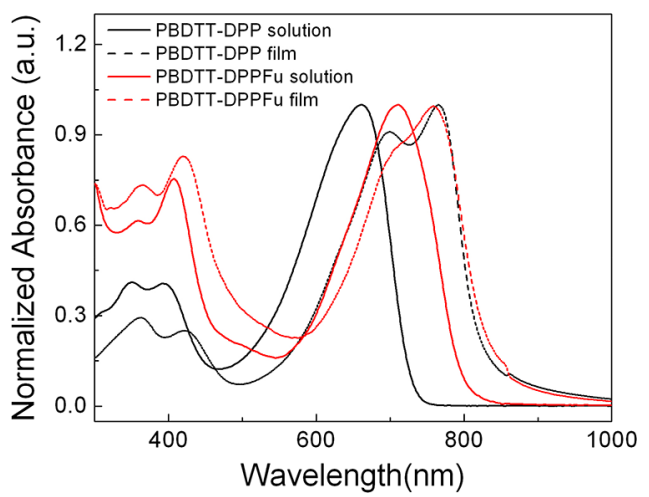

Figure 1. Normalized absorption spectra of the two polymers (solid: in chloroform; dashed: in film). absorption bands, which can be attributed to manifold $\pi-\pi^{*}$ transitions and are primarily originated from local excitations and/or intramolecular charge-transfer excitations. In chloroform solution, the maximum absorption wavelength $\left(\lambda_{\max }\right)$ of PBDTT-DPPFu at $710 \mathrm{~nm}$ is redshifted by $50 \mathrm{~nm}$ as compared to that of PBDTT-DPP at $660 \mathrm{~nm}$. In contrast, both polymers show a similar $\lambda_{\text {max }}$ located at $\sim 760 \mathrm{~nm}$ when spin-coated as films, suggesting that both polymers have good backbone planarity and $\pi-\pi$ stacking in the solid state and that in solution, PBDTT-DPP may assume more twisted backbone conformations originated from the single bond rotation freedom within the acceptor segments, which is not possible after ring-fusion. This, in conjunction with the abovementioned molecular weight difference of the two polymers, indicates that the backbone of PBDTT-DPPFu exhibits relatively planar conformation in solution due to the enforcement of the molecular coplanarity by ring-fusion. It is worth noting that PBDTT-DPPFu presents redshifted absorption by $19 \mathrm{~nm}$ at maximum and much broader absorption as compared to its analogue PEDDPT $_{\mathrm{HD}^{-B D T}}$ (steep absorption edges and small Stokes shifts), which is probably due to the use of dialkyl thiophenes as side groups on BDT units instead of alkoxyl side chains. ${ }^{40}$

We then investigate the influence of the ring-fusion effect on the frontier energy levels of the copolymer. The energy levels and energy gaps of polymers are estimated from their corresponding redox potentials by cyclic voltammetry $(\mathrm{CV})$ measurements. Figure 2 shows the cyclic voltammograms of

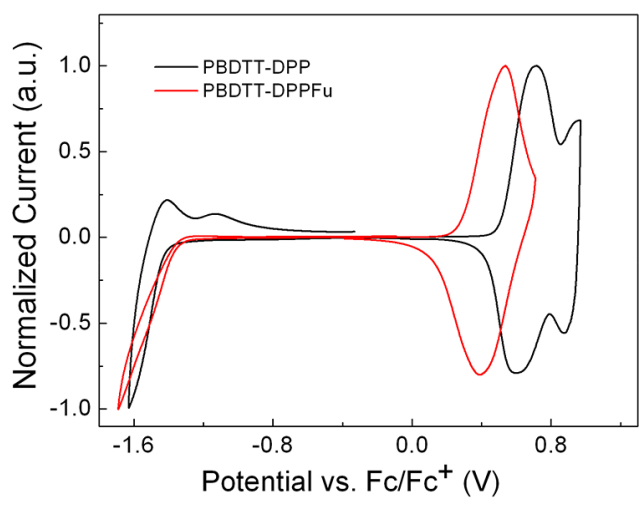

Figure 2. Cyclic voltammetry (CV) curves of the two polymers.

the two polymers. According to the equations HOMO $=-\left(E_{\mathrm{ox}}\right.$ $+5.13) \mathrm{eV}$ and LUMO $=-\left(E_{\mathrm{red}}+5.13\right) \mathrm{eV},{ }^{41-43}$ the HOMO levels of PBDTT-DPP and PBDTT-DPPFu are estimated to be -5.66 and $-5.50 \mathrm{eV}$ and the LUMO levels are -3.97 and $-3.88 \mathrm{eV}$, respectively. According to the equation, $E_{\mathrm{g}}{ }^{\mathrm{ec}}=$ LUMO - HOMO, the electrochemical energy gaps of the two polymers are estimated to be 1.69 and $1.62 \mathrm{eV}$ for PBDTTDPP and PBDTT-DPPFu, respectively. It is interesting to note that when going from PBDTT-DPP to PBDTT-DPPFu, both HOMO and LUMO levels upshift with the HOMO level 

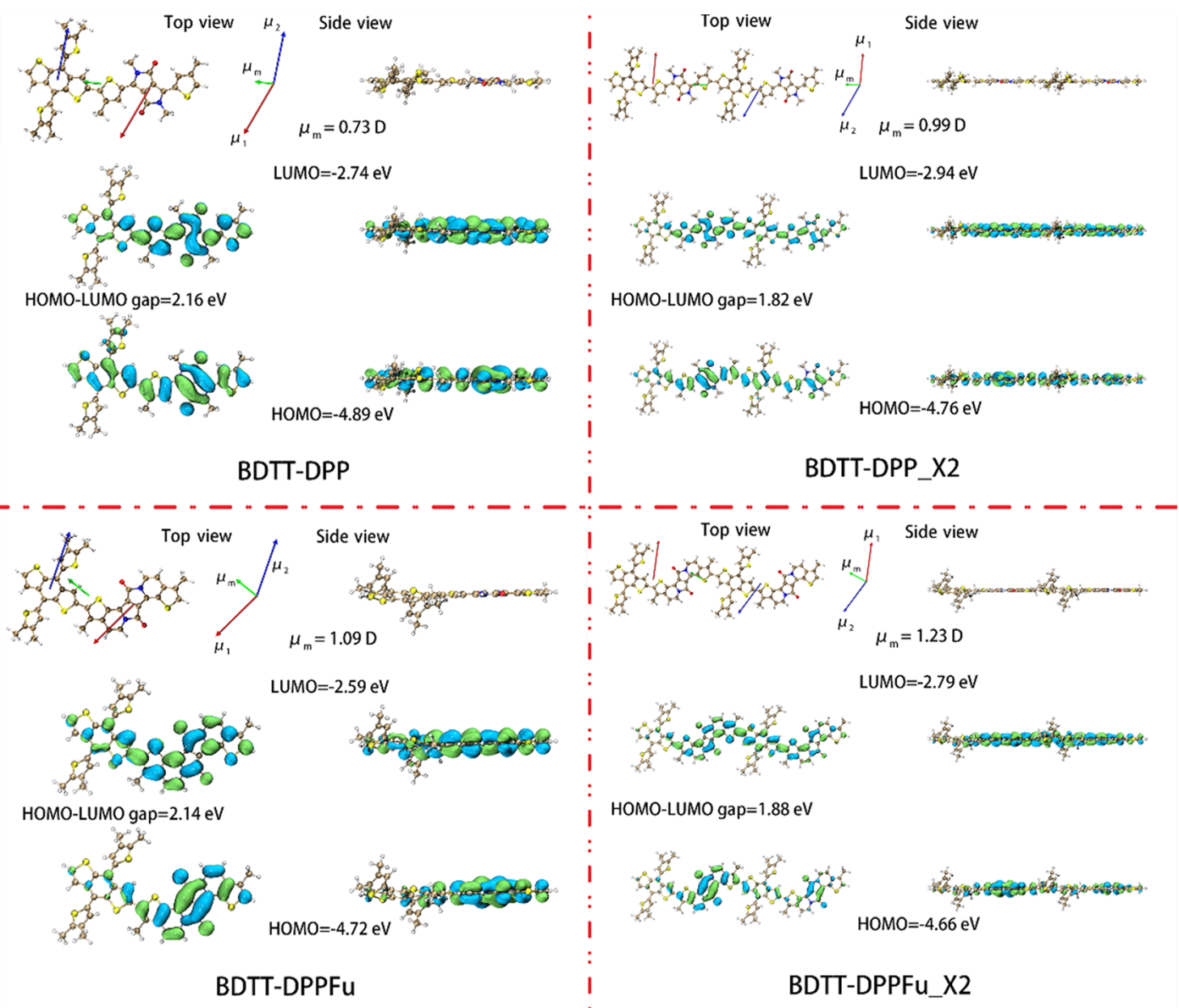

Figure 3. Optimized geometries, frontier molecular orbitals (isovalue: 0.02 a.u.), and molecular dipole moments.

shifted in a larger extent, resulting in a slightly narrowed energy gap for PBDTT-DPPFu. The electrochemical gaps follow the same trend as optical gaps deduced from onsets of the solidstate absorption.

Theoretical Calculations. To gain insight into how ringfusion can affect the backbone of the conjugated molecules, DFT and TD-DFT calculations via an oligomer approach were performed on the two synthesized conjugated polymers (PBDTT-DPP and PBDTT-DPPFu) for comparison in the gas phase. Computations were performed on the models based on one and two repeating units for comparison. The bulky alkyl or alkoxyalkyl side chains are simplified to methyl groups. The geometries were optimized at the B3LYP-D3(BJ)/def2SVP level. ${ }^{4-46}$ It is found that without bulky side chains, the two backbone molecules show almost comparable backbone coplanarity, the main difference of which lies in the DAD segment of the PBDTT-DPP and the fused acceptor unit of the PBDTT-DPPFu. If there are no sterically hindering side chains attached, it is possible for both backbones to assume much more planar coplanarity upon solid-state stacking. However, when bulky side chains are installed, the backbone coplanarity of the fused backbones can be better guaranteed than that of the unfused one due to ring-fusion-promoted backbone rigidity.

It has to be noted that for the PBDTT-DPP polymer without ring-fusion, the DPP unit may assume different conformations originated from the single bond rotation freedom between the acceptor core and the flanking thiophene units, for example, if forced by steric hindrance caused by bulky side chains attached, while this is not possible for the PBDTTDPPFu with ring-fusion in the acceptor segments. To understand how this can affect the properties of the resulting polymers, two conformations of the DPP units in the tworepeating-unit model of PBDTT-DPP polymer were calculated at the same level of theory (Figure $S 1$ and Table S1). It is found that PBDTT-DPP is dominated by the conformer PBDTT-DPPa, which is $8.05 \mathrm{kcal} \mathrm{mol}^{-1}$ lower in Gibbs free energy in the gas phase and is $3.50 \mathrm{kcal} \mathrm{mol}^{-1}$ lower in Gibbs free energy in chloroform solution. According to the Boltzmann distribution law, at room temperature, the Boltzmann population of the predominant conformer PBDTT-DPPa in both cases will be over 99\%. The conformational effect on the calculated HOMO and LUMO energies cannot be neglected especially if there is influence from bulky side chains. The HOMO and LUMO energies are calculated to be -4.76 and $-2.94 \mathrm{eV}$ for PBDTT-DPP $a$ and -4.74 and $-2.86 \mathrm{eV}$ for PBDTT-DPP $b$, respectively, at the B3LYP-D3(BJ)/def2-SVP level, corresponding to a HOMOLUMO gap of $1.82-1.89 \mathrm{eV}$. For PBDTT-DPPFu with ringfusion, the HOMO, LUMO, and HOMO-LUMO gap energies are $-4.66,-2.79$, and $1.87 \mathrm{eV}$, respectively. It is noted that ring-fusion of the DAD segment results in upshifted HOMO and LUMO energy levels as also observed by electrochemical measurements. Theoretically, it would be expected for PBDTT-DPP to have a more narrowed energy gap than PBDTT-DPPFu, which is contrary to the experimental results 


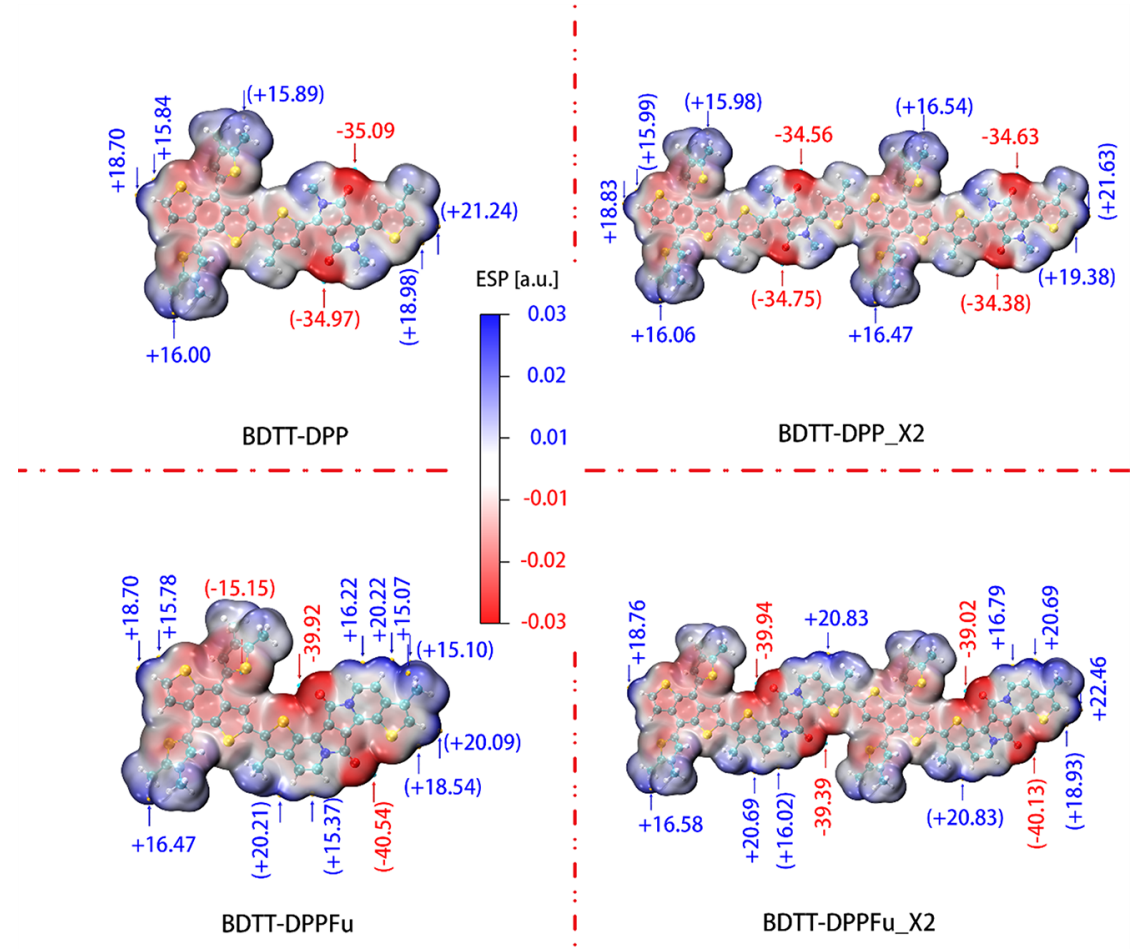

Figure 4. ESP isosurface map (isovalue: 0.001 a.u.). The small orange and cyan spheres correspond to the most positive and negative points, respectively, which are labeled in $\mathrm{kcal} \mathrm{mol}^{-1}$. The numbers in parentheses indicate that the spheres are located in the backside of the view.

obtained by optical and electrochemical measurements. This may infer that unlike PBDTT-DPPFu, PBDTT-DPP may suffer from a decrease in backbone planarity and/or stacking order or have a steric hindrance-driven conformational preference due to the rotation freedom of the single bonds between the acceptor core and flanking thiophenes when bulky side chains are used. Nevertheless, unless otherwise specified, the energetically favored PBDTT-DPP $a$ will be still taken as a representative conformer of PBDTT-DPP for later discussion. ${ }^{41-43}$ Additionally, it should be mentioned that the simplification of the diethoxy ethyl side group as the methyl group in the calculation model for computational cost is reasonable since the oxygen atoms in the side chains are not directly bonded to the backbone and should have limited influence on the photophysical properties of the polymer. Calculations (Figure S1) show that the ether side groups on the DPP units of the PBDTT-DPP polymer slightly lower the HOMO and LUMO levels simultaneously by around $0.05 \mathrm{eV}$ while keeping the HOMO-LUMO gaps almost unchanged as compared to the counterpart with methyl side chains. Note that by comparing these values to their counterparts obtained under the same conditions for the one-repeating-unit model compounds, the energy gaps of the ring-fused structures narrow with extending the conjugation backbones less significantly. This holds also true when we look at the vertical excitation energy as is discussed later.

For all the molecules, both HOMOs and LUMOs are delocalized over the whole backbones (Figure 3), but ringfusion on the acceptor units caused the HOMO wave function to partially localize more to these acceptor units while the distribution of LUMOs are almost unchanged, which can be accountable for the change in energy levels and gaps. To further understand these differences and to compare their orbital delocalization extent, we calculated their orbital delocalization index (ODI) based on the Hirshfeld method. $^{47,48}$ The ODI is an indicator of quantifying the extent of orbital spatial delocalization, calculated by the Multiwfn program. The smaller ODI value means that the orbitals are spatially more distributed to more atoms within a molecule and are therefore deemed as being more delocalized. The ODI versus molecular orbital index of each structure of occupied orbitals (from HOMO down to HOMO-10) was plotted for comparison (Figure S2). Indeed, it is found that after ringfusion of the molecular structure, the HOMOs of BDTTDPPFu series have slightly higher ODI values and are therefore less delocalized than those of BDTT-DPP series based on the same number of repeating units, while the LUMOs of the ringfused BDTT-DPPFu series are more delocalized than their unfused counterparts.

Intermolecular dipole interactions between conjugated molecules can drive neighboring molecules to form aggregates, particularly when the chromophore possesses a large dipole moment. ${ }^{49}$ To evaluate the molecular dipole moments of the studied structures, single point calculations using a Karlsruhe split-valence basis set def2-SVPD ${ }^{46,50}$ with polarization and diffuse functions were performed based on the B3LYPD3(BJ)/def2-SVP optimized geometry. Calculated data (Figure 3) show that ring-fusion of PBDTT-DPP results in a small increase in the dipole moments over the whole molecules, which is expected to favor charge transport through enhanced intermolecular interactions.

Usually, the electrostatic interaction plays a crucial role among the molecular interactions between the system under study and external environment. The isosurface map of electrostatic potential (ESP) on the van der Waals (vdW) surface is investigated since it is very closely related to intermolecular electrostatic interactions, to gain information of molecular properties and intermolecular interactions. ${ }^{51}$ The 


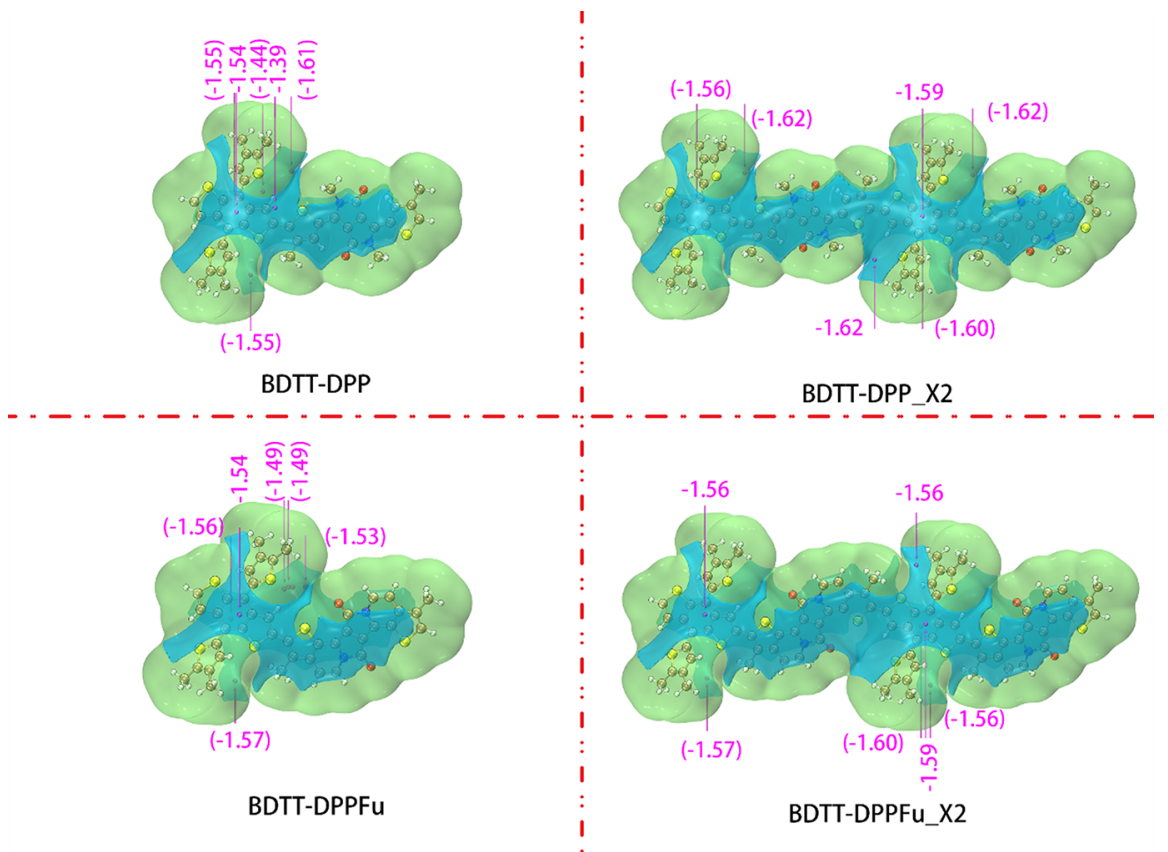

Figure 5. Isosurface map of van der Waals potentials (isovalue: 1.0). Green and blue isosurfaces correspond to positive and negative distributions, respectively. The small magenta spheres correspond to the most negative points, which are labeled in $\mathrm{kcal} \mathrm{mol}^{-1}$. The numbers in parentheses indicate that the spheres are located in the backside of the view.

ESP maxima and minima on the vdW surface are also calculated (Figure 4). It can be seen that the fused system has a greater electronegativity difference over the unfused system, for example, the ESP on the vdW surface ranges from -34.75 to $21.63 \mathrm{kcal} \mathrm{mol}^{-1}$ for PBDTT-DPP (BDTT-DPP_X2) and from -40.13 to $22.46 \mathrm{kcal} \mathrm{mol}^{-1}$ for PBDTT-DPPFu (BDTT$\mathrm{DPPFu}$ X2). In these structures, oxygen has a higher electronegativity than sulfur and other elements, and therefore, oxygen atoms would consequently have a higher electron density around them than other atoms. The ESP analysis results show that the oxygen atoms of the amide group on the acceptor units have the most negative surface potential, while $\mathrm{C}-\mathrm{H}$ adjacent to $\mathrm{S}$ of thiophene rings have the most positive surface potential. Additionally, it can be seen that the bridges introduced by ring-fusion of the acceptor units in this study also have significant positive surface potential. Meanwhile, due to the change in the backbone configuration after ring-fusion, the thiophene side groups become facing the amide oxygen negative surface potential regions, different from the unfused system. Consequently, the fused system has a greater electronegativity difference over the unfused system and can form a stronger electrostatic interaction to other molecules, enhancing their intermolecular interaction with other molecules. The ESP analysis results agree well with the DFT calculations on the more localization of HOMOs on the acceptor units for the fused systems.

Another type of molecular interaction known as van der Waals interaction can sometimes play important roles under some circumstances, for example, when interacting molecules are nonpolar or weakly polar. The trade-off between the exchange-repulsion interaction showing a repulsive effect and the dispersion interaction showing an attractive effect will result in different intermolecular interaction behavior. The isosurface map of the vdW potential evaluated by the LennardJones 12-6 potential shows that the regions (blue isosurface) where the dispersion attraction effect surpasses the exchange- repulsion effect are favorable physical adsorption zones for intermolecular packing (Figure 5). ${ }^{48,52}$ Similarly, in all cases, these regions are mainly facing to the plane of the conjugated backbones, beneficial to $\pi-\pi$ stacking, and the most favorable adsorption sites are close to the donor units of the conjugated backbones. Ring-fusion of the acceptor units does not seem to cause much difference in the vdW interaction strength. From the above discussion of molecular dipole moments, electrostatic interactions, and van der Waals interactions, it is expected that ring-fusion will enhance the intermolecular interaction, molecular packing, and as a result the electronic properties of these conjugated systems.

To assess the excited-state vertical excitation energies and oscillator strengths of the systems, we further performed timedependent DFT calculations with range-separated hybrid functionals at the CAM-B3LYP-D3(BJ)/def2-SVP level ${ }^{45,46,53}$ based on the geometry optimized at the B3LYP-D3(BJ)/def2SVP level. The major contributions of molecular orbitals to the electronic transitions are also summarized in Table S2. TDDFT calculation reveals that the lowest excitations from the ground state $\left(S_{0}\right)$ to the first excited state $\left(S_{1}\right)$ correspond to $\pi-\pi^{*}$ transitions and are all dominated by the HOMO $\rightarrow$ LUMO transitions. The excited-state vertical transition energies show similar trends to the HOMO-LUMO gaps among the studied structures. It can be found that the oscillator strengths of the fused systems are relatively lower than those of the unfused PBDTT-DPP especially in the case of the $S_{0} \rightarrow S_{1}$ transitions. According to Kasha's rule, the first excited state of a singlet system is usually the critical state to emit fluorescence and hence plays an important role in molecular photophysics. From the molecular orbital contribution to the transitions, it can be seen that most of the transitions of the system studied cannot be simply expressed as a transition between a specific pair of molecular orbitals but a consequence of combined contributions from several pairs of molecular orbitals. 
Table 2. OFET Mobilities for PBDTT-DPP and PBDTT-DPPFu Using Different Dielectric Layers and Summarized GIWAXS Data

\begin{tabular}{|c|c|c|c|c|c|c|}
\hline \multirow[b]{2}{*}{ polymers } & \multicolumn{2}{|c|}{ PMMA } & \multirow{2}{*}{$\frac{\mathrm{PTrFE}}{\mu_{\mathrm{h}}^{a}\left(\mathrm{~cm}^{2} \mathrm{~V}^{-1} \mathrm{~s}^{-1}\right)}$} & \multirow{2}{*}{$\frac{\text { spacing } d_{010}(\AA)}{\pi-\pi \text { stacking }}$} & \multicolumn{2}{|c|}{ coherence length $L_{c}(\AA)$} \\
\hline & $\mu_{\mathrm{h}}^{a}\left(\mathrm{~cm}^{2} \mathrm{~V}^{-1} \mathrm{~s}^{-1}\right)$ & $\mu_{\mathrm{e}}^{a}\left(\mathrm{~cm}^{2} \mathrm{~V}^{-1} \mathrm{~s}^{-1}\right)$ & & & OOP & IP \\
\hline PBDTT-DPP & $(1.2 \pm 0.60) \times 10^{-4}$ & $(4.1 \pm 0.26) \times 10^{-3}$ & $(6.0 \pm 0.12) \times 10^{-4}$ & 4.19 & 12.2 & 11.4 \\
\hline PBDTT-DPPFu & $(1.8 \pm 0.10) \times 10^{-3}$ & $(1.0 \pm 0.15) \times 10^{-2}$ & $(2.5 \pm 0.65) \times 10^{-3}$ & 4.19 & 16.2 & 17.2 \\
\hline
\end{tabular}

${ }^{a}$ The FET mobilities were calculated in the saturation regime $\left(V_{\mathrm{D}}= \pm 100 \mathrm{~V}\right)$.

Hence, electron-hole analysis ${ }^{48,54}$ was carried out by the Multiwfn program to gain insight into their underlying characteristics such as how charge transfer takes place along the backbones. There are several quantities to be calculated and discussed. The $H$ index is an overall measure of the average spatial extension degree of hole and electron distribution based on their root-mean-square deviations (RMSDs). The $D$ index is the total magnitude of chargetransfer (CT) length as measured by the distance between the centers of mass of electrons and holes. The $t$ index represents the difference between the $D$ index and the average spatial extension degree of hole and electron distribution in the CT direction, characterizing the separation degree of holes and electrons in the CT direction, with negative values implying that the holes and electrons are not substantially separated due to CT. The $S_{\mathrm{r}}$ index is a measure to characterize the overlapping extent of holes and electrons by integration of the geometric mean of their charge densities over all space. Generally, the higher $H$ index value in the hole-electron analysis, the better distribution along the entire backbone on average. As can be seen from Table S3 and Figure S3 (Supporting Information), for these conjugated systems, electrons and holes of different excited states are on average distributed and delocalized over the entire backbones, as indicated by relatively high $H$ index values (for average overall distribution of electrons and holes) as well as low hole delocalization index (HDI) and electron delocalization index (EDI) values. The electrons and holes are very close to each other and highly overlapped, with the $D$ index (center of mass distance of electron and hole) generally less than one bond length and the $S_{\mathrm{r}}$ index (extent of electron-hole overlap) ranging from over 50 up to $87 \%$, and therefore, most of the transitions can be deemed as local excitation of highly localized $\pi-\pi^{*}$ nature. In all cases, the negative values of all the $t$ index also confirm that the holes and electrons are not significantly separated relative to their good distribution though, in accord with the $D$ index. The Coulomb attractive energies $\left(E_{\mathrm{C}}\right)$ between holes and electrons of all excited states studied are basically lower than $4.86 \mathrm{eV}$, implying that the holes and electrons can be either well separated or distributed. It is worth mentioning that the $S_{0} \rightarrow S_{1}$ transitions have the highest transition dipole moments and oscillator strengths compared to other transitions of the same systems. A higher overlap of well-distributed holes and electrons of the excited states may favor a higher oscillator strength of the corresponding transition, for example, as a consequence of a combination of a higher $S_{\mathrm{r}}$ index and a lower $D$ index along with a higher extended conjugation path length.

OFET Performance. In order to characterize the charge transport properties of these copolymers, top-gate bottomcontact OFETs were fabricated. Poly(methyl methacrylate) (PMMA) with a thickness of $\sim 600 \mathrm{~nm}$ was used as a dielectric layer. A detailed description of the fabrication process is given in the Supporting Information. Interestingly, all tested devices that include PMMA as the dielectric layer based on PBDTTDPP and PBDTT-DPPFu exhibit ambipolar characteristics under an ambient atmosphere. All the mobility values were calculated in the saturation region $\left(V_{\mathrm{D}}= \pm 100 \mathrm{~V}\right)$, as shown in Table 2. For PBDTT-DPP, a hole mobility of $(1.2 \pm 0.60) \times$ $10^{-4} \mathrm{~cm}^{2} \mathrm{~V}^{-1} \mathrm{~s}^{-1}$ and an electron mobility of $(4.1 \pm 0.26) \times$ $10^{-3} \mathrm{~cm}^{2} \mathrm{~V}^{-1} \mathrm{~s}^{-1}$ were obtained (Figure 6a). Remarkably, the
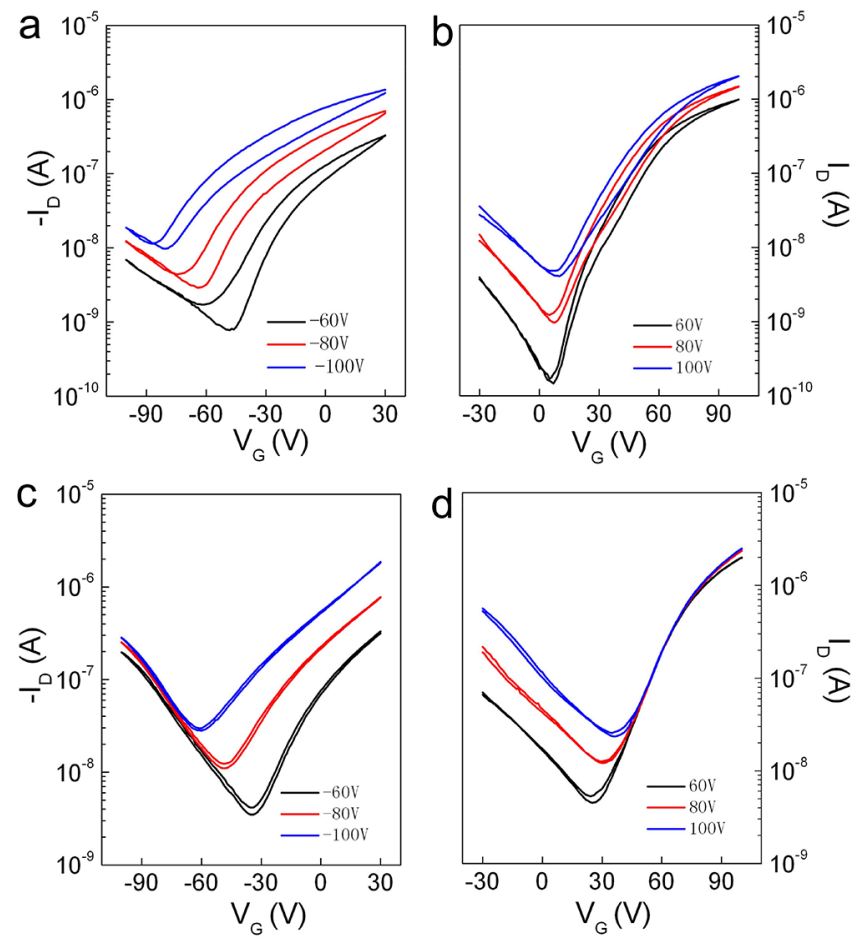

Figure 6. Transfer curves of OFETs using PMMA as a dielectric layer based on polymer films of (a, b) PBDTT-DPP and (c, d) PBDTTDPPFu.

hole mobility of PBDTT-DPPFu is about one order of magnitude higher $\left((1.8 \pm 0.10) \times 10^{-3} \mathrm{~cm}^{2} \mathrm{~V}^{-1} \mathrm{~s}^{-1}\right)$ as compared to PBDTT-DPP, whereas the electron mobility undergoes a 2 -fold increase $\left((1.0 \pm 0.15) \times 10^{-2} \mathrm{~cm}^{2} \mathrm{~V}^{-1} \mathrm{~s}^{-1}\right)$ (Figure 6c). Such an enhancement can be attributed to the strong molecular interactions of PBDTT-DPPFu, leading to better $\pi-\pi$ stacking and thus a higher charge carrier mobility.

High- $k$ fluorinated polymers such as poly(trifluoroethylene) (PTrFE, Solvay S.A.) were also used as the dielectric layer in the OFETs. In fact, it has been shown that high- $k$ fluorinated dielectrics enhance hole transport in polymeric OFETs, which then gives us the possibility to improve the mobility values. $^{28,55-57}$ Interestingly, hole mobility values as high as $(6.0 \pm 0.12) \times 10^{-4}$ and $(2.5 \pm 0.65) \times 10^{-3} \mathrm{~cm}^{2} \mathrm{~V}^{-1} \mathrm{~s}^{-1}$ are detected for PBDTT-DPP and PBDTT-DPPFu, respectively (Table 2 and Figure 7). Noteworthily, these hole mobility values are higher than those attained using PMMA as a 

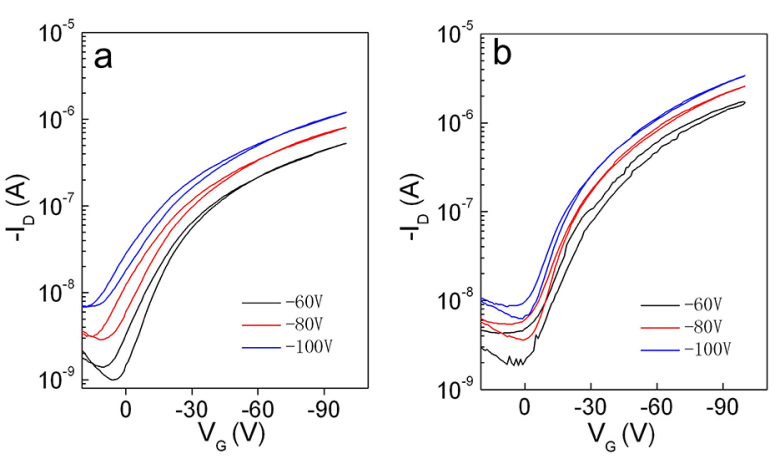

Figure 7. Transfer curves of OFETs using PTrFE as a dielectric layer based on polymer films of (a) PBDTT-DPP and (b) PBDTT-DPPFu.

dielectric layer. However, no electron mobility can be detected due to the suppression of electron transport, which is induced by the high- $k$ fluorinated dielectrics. Thus, the devices show unipolar transport behavior (Figure 7). Nevertheless, the effect of ring-fusion can still be clearly seen in the PTrFE devices. It should be noted that the molecular weight of PBDTT-DPPFu $\left(M_{\mathrm{n}}=17 \mathrm{kDa}\right)$ is much lower than that of PBDTT-DPP $\left(M_{\mathrm{n}}=\right.$ $108 \mathrm{kDa})$. Generally, a relatively higher molecular weight gives higher mobility values for the same polymer structure due to less defects and extended polymer chains. ${ }^{11,12}$ However, our results indicate that ring-fusion-induced molecular packing improves the field-effect mobility and thus the device performance, to a relatively greater extent than any effects related to polymeric molecular weight.

To gain insight into the correlation between molecular packing and the device performance, we investigated the polymer film microstructure by grazing incidence wide-angle X-ray scattering (GIWAXS). Figure $8 \mathrm{a}, \mathrm{b}$ shows the GIWAXS
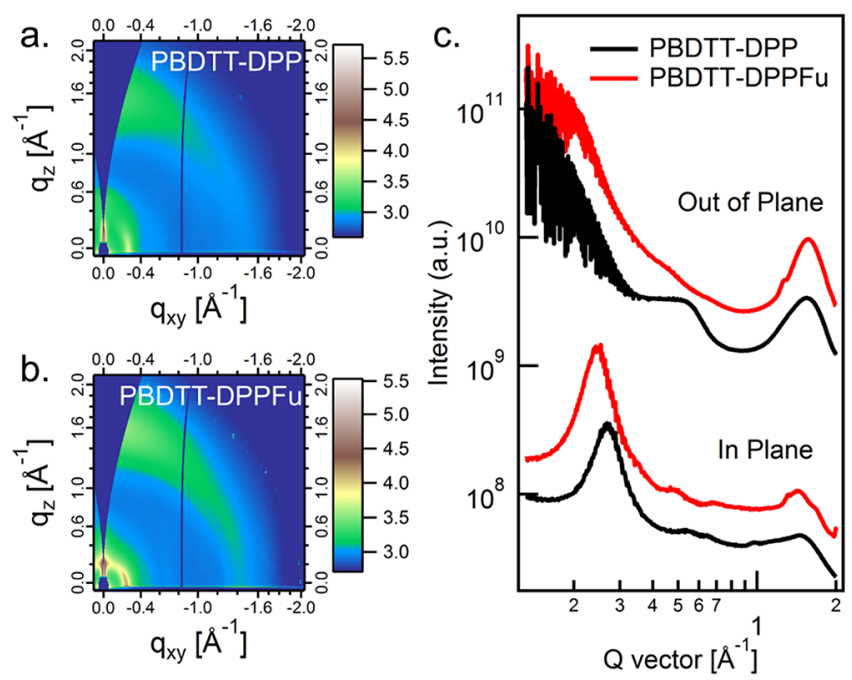

Figure 8. Grazing incidence X-ray scattering $2 \mathrm{D}$ patterns of (a) PBDTT-DPP and (b) PBDTT-DPPFu. (c) Corresponding GIWAXS profiles of copolymer films.

two-dimensional patterns of PBDTT-DPP and PBDTT$\mathrm{DPPFu}$ with corresponding profiles reported in Figure 8c. Both PBDTT-DPP and PBDTT-DPPFu show obvious (100) peaks in the in-plane (IP) direction and (010) peaks in the out-of-plane (OOP) direction as revealed in Figure 8a,b, respectively, which indicates a face-on orientation preference. $\pi-\pi$ stacking resembles the $(010)$ peaks at $q \approx 1.5 \AA^{-1}$ and is critical to charge transport. The corresponding calculated coherence length values are 12.2 and 16.2 $\AA$ for PBDTT-DPP and PBDTT-DPPFu in the out-of-plane direction and 11.4 and 17.2 $\AA$ in the in-plane direction, respectively. This indicates a relatively stronger interaction between the neighboring molecules of PBDTT-DPPFu as compared to PBDTT-DPP. Considering the much lower molecular weight of PBDTT$\operatorname{DPPFu}\left(M_{\mathrm{n}}=17 \mathrm{kDa}\right.$ for PBDTT-DPPFu and $M_{\mathrm{n}}=108 \mathrm{kDa}$ for PBDTT-DPP), we can attribute the enhanced mobility in PBDTT-DPPFu to its better molecular stacking.

\section{CONCLUSIONS}

In conclusion, we have successfully synthesized and characterized ring-fused DPP-based copolymers. It is found that ringfusion of the DPP copolymer increases the intermolecular interactions of the DPP copolymer, which is confirmed by UV-vis spectroscopy, DFT calculation, ESP and vdW analyses, as well as XRD data. As a consequence, the ringfused DPP copolymer shows improved charge carrier mobility even with much lower molecular weight. Higher performance can be expected when the molecular weight of the polymer is further increased. The results from our comparative study provide a robust way to increase the interchain interaction by ring-fusion methods. Our results highlighted the ring-fused DPP unit as a promising building block for construction of high-performance polymers for organic electronics.

\section{ASSOCIATED CONTENT}

\section{SI Supporting Information}

The Supporting Information is available free of charge at https://pubs.acs.org/doi/10.1021/acs.macromol.0c02326.

Experimental details, synthesis of monomers and polymers, details for theoretical calculations and additional data of DFT/TD-DFT calculations and wave function analysis, details for OFET fabrication and characterization, GPC curves, and NMR spectra (PDF)

\section{AUTHOR INFORMATION}

\section{Corresponding Authors}

Simone Fabiano - Laboratory of Organic Electronics, Department of Science and Technology, Linköping University, SE-60174 Norrköping, Sweden; 이이.org/0000-00017016-6514; Email: simone.fabiano@liu.se

Weiguo Zhu - School of Materials Science and Engineering, Jiangsu Collaborative Innovation Center of Photovoltaic Science and Engineering, Changzhou University, Changzhou 213164, China; 이이.org/0000-0002-4244-2638; Email: zhuwg18@126.com

Ergang Wang - Department of Chemistry and Chemical Engineering, Chalmers University of Technology, SE-41296 Göteborg, Sweden; School of Materials Science and Engineering, Zhengzhou University, Zhengzhou 450001,

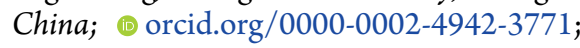

Email: ergang@chalmers.se

\section{Authors}

Wenliu Zhuang - Advanced Research Center for Polymer Processing Engineering of Guangdong Province, Guangdong Industry Polytechnic, Guangzhou 510300, China; Department of Chemistry and Chemical Engineering, Chalmers University of Technology, SE-41296 Göteborg, Sweden; ㅇo이.org/0000-0003-3159-756X 
Suhao Wang - Laboratory of Organic Electronics, Department of Science and Technology, Linköping University, SE-60174 Norrköping, Sweden

Qiang Tao - Department of Chemistry and Chemical Engineering, Chalmers University of Technology, SE-41296 Göteborg, Sweden; School of Materials and Chemical Engineering, Hunan Institute of Engineering, Xiangtan 411104, China

Wei Ma - State Key Laboratory for Mechanical Behavior of Materials, Xi'an Jiaotong University, Xi'an 710049, China; () orcid.org/0000-0002-7239-2010

Magnus Berggren - Laboratory of Organic Electronics, Department of Science and Technology, Linköping University, SE-60174 Norrköping, Sweden

Complete contact information is available at:

https://pubs.acs.org/10.1021/acs.macromol.0c02326

\section{Author Contributions}

${ }^{\ddagger}$ W. Zhuang. S.W., and Q.T. contributed equally to this work. The manuscript was written through contributions of all authors. All authors have given approval to the final version of the manuscript.

\section{Notes}

The authors declare no competing financial interest.

\section{ACKNOWLEDGMENTS}

We thank the Swedish Research Council (2015-04853, 201606146, and 2019-04683), the Swedish Research Council Formas, and NSFC (21504006 and 21534003) for financial support. Further, the authors also wish to acknowledge the financial support from the Knut and Alice Wallenberg Foundation (2017.0186 and 2016.0059) and the Swedish Foundation for Strategic Research. W. Zhuang. appreciates support from the China Scholarship Council (201908440047), NSFC project (11272093), Guangzhou Municipal Science and Technology Bureau (201804010501, 201904010381, and 202002030362), Department of Education of Guangdong Province (2018GKTSCX041 and 2017GKQNCX005), Department of Science and Technology of Guangdong Province (2016A010103046), and the Open Fund of the State Key Laboratory of Luminescent Materials and Devices (South China University of Technology, 2020-skllmd-07). Q.T. acknowledges the support from the Natural Science Foundation of Hunan Province (2018JJ3098). X-ray data were acquired at beamline 7.3.3 at the Advanced Light Source, which is supported by the Director, Office of Science, Office of Basic Energy Sciences, of the U.S. Department of Energy under contract no. DE-AC02-05CH11231. The quantum chemical computations were performed on resources provided by the Swedish National Infrastructure for Computing (SNIC) at $\mathrm{C}^{3} \mathrm{SE}$ in Gothenburg.

\section{REFERENCES}

(1) Berggren, M.; Nilsson, D.; Robinson, N. D. Organic materials for printed electronics. Nat. Mater. 2007, 6, 3-5.

(2) Knopfmacher, O.; Hammock, M. L.; Appleton, A. L.; Schwartz, G.; Mei, J.; Lei, T.; Pei, J.; Bao, Z. Highly stable organic polymer fieldeffect transistor sensor for selective detection in the marine environment. Nat. Commun. 2014, 5, 2954.

(3) Kim, G.; Kang, S. J.; Dutta, G. K.; Han, Y. K.; Shin, T. J.; Noh, Y. Y.; Yang, C. A Thienoisoindigo-Naphthalene Polymer with Ultrahigh Mobility of $14.4 \mathrm{~cm}(2) /$ V.s That Substantially Exceeds Benchmark
Values for Amorphous Silicon Semiconductors. J. Am. Chem. Soc. 2014, 136, 9477-9483.

(4) Tseng, H. R.; Phan, H.; Luo, C.; Wang, M.; Perez, L. A.; Patel, S. N.; Ying, L.; Kramer, E. J.; Nguyen, T. Q.; Bazan, G. C.; Heeger, A. J. High-Mobility Field-Effect Transistors Fabricated with Macroscopic Aligned Semiconducting Polymers. Adv. Mater. 2014, 26, 2993-2998.

(5) Gao, Y.; Zhang, X.; Tian, H.; Zhang, J.; Yan, D.; Geng, Y.; Wang, F. High Mobility Ambipolar Diketopyrrolopyrrole-Based Conjugated Polymer Synthesized Via Direct Arylation Polycondensation. Adv. Mater. 2015, 27, 6753-6759.

(6) Yamashita, Y.; Hinkel, F.; Marszalek, T.; Zajaczkowski, W.; Pisula, W.; Baumgarten, M.; Matsui, H.; Müllen, K.; Takeya, J. Mobility Exceeding $10 \mathrm{~cm} 2 /(\mathrm{V} \cdot \mathrm{s})$ in Donor-Acceptor Polymer Transistors with Band-like Charge Transport. Chem. Mater. 2016, 28, 420-424.

(7) Back, J. Y.; Yu, H.; Song, I.; Kang, I.; Ahn, H.; Shin, T. J.; Kwon, S. K.; Oh, J. H.; Kim, Y. H. Investigation of Structure-Property Relationships in Diketopyrrolopyrrole-Based Polymer Semiconductors via Side-Chain Engineering. Chem. Mater. 2015, 27, 1732-1739.

(8) Yi, Z.; Wang, S.; Liu, Y. Design of High-Mobility Diketopyrrolopyrrole-Based pi-Conjugated Copolymers for Organic Thin-Film Transistors. Adv. Mater. 2015, 27, 3589-3606.

(9) Liu, Q.; Bottle, S. E.; Sonar, P. Developments of Diketopyrrolopyrrole-Dye-Based Organic Semiconductors for a Wide Range of Applications in Electronics. Adv. Mater. 2020, 32, 1903882.

(10) McCulloch, I.; Heeney, M.; Bailey, C.; Genevicius, K.; MacDonald, I.; Shkunov, M.; Sparrowe, D.; Tierney, S.; Wagner, R.; Zhang, W.; Chabinyc, M. L.; Kline, R. J.; McGehee, M. D.; Toney, M. F. Liquid-crystalline semiconducting polymers with high chargecarrier mobility. Nat. Mater. 2006, 5, 328-333.

(11) Müller, C.; Wang, E.; Andersson, L. M.; Tvingstedt, K.; Zhou, Y.; Andersson, M. R.; Inganäs, O. Influence of Molecular Weight on the Performance of Organic Solar Cells Based on a Fluorene Derivative. Adv. Funct. Mater. 2010, 20, 2124-2131.

(12) Tsao, H. N.; Cho, D. M.; Park, I.; Hansen, M. R.; Mavrinskiy, A.; Yoon, D. Y.; Graf, R.; Pisula, W.; Spiess, H. W.; Müllen, K. Ultrahigh Mobility in Polymer Field-Effect Transistors by Design. J. Am. Chem. Soc. 2011, 133, 2605-2612.

(13) Fabiano, S.; Musumeci, C.; Chen, Z.; Scandurra, A.; Wang, H.; Loo, Y. L.; Facchetti, A.; Pignataro, B. From monolayer to multilayer $\mathrm{N}$-channel polymeric field-effect transistors with precise conformational order. Adv. Mater. 2012, 24, 951-956.

(14) Tseng, H.-R.; Ying, L.; Hsu, B. B.; Perez, L. A.; Takacs, C. J.; Bazan, G. C.; Heeger, A. J. High mobility field effect transistors based on macroscopically oriented regioregular copolymers. Nano Lett. 2012, 12, 6353-6357.

(15) Wang, S.; Kiersnowski, A.; Pisula, W.; Müllen, K. Microstructure evolution and device performance in solution-processed polymeric field-effect transistors: the key role of the first monolayer. J. Am. Chem. Soc. 2012, 134, 4015-4018.

(16) Wang, S.; Pisula, W.; Müllen, K. Nanofiber growth and alignment in solution processed $\mathrm{n}$-type naphthalene-diimide-based polymeric field-effect transistors. J. Mater. Chem. 2012, 22, 2482724831.

(17) Fabiano, S.; Yoshida, H.; Chen, Z.; Facchetti, A.; Loi, M. A. Orientation-dependent electronic structures and charge transport mechanisms in ultrathin polymeric n-channel field-effect transistors. ACS Appl. Mater. Interfaces 2013, 5, 4417-4422.

(18) Luo, C.; Kyaw, A. K. K.; Perez, L. A.; Patel, S.; Wang, M.; Grimm, B.; Bazan, G. C.; Kramer, E. J.; Heeger, A. J. General strategy for self-assembly of highly oriented nanocrystalline semiconducting polymers with high mobility. Nano Lett. 2014, 14, 2764-2771.

(19) Tremel, K.; Fischer, F. S. U.; Kayunkid, N.; Di Pietro, R.; Tkachov, R.; Kiriy, A.; Neher, D.; Ludwigs, S.; Brinkmann, M. Charge Transport Anisotropy in Highly Oriented Thin Films of the Acceptor Polymer P(NDI2OD-T2). Adv. Energy Mater. 2014, 4, 1301659.

(20) Bucella, S. G.; Luzio, A.; Gann, E.; Thomsen, L.; McNeill, C. R.; Pace, G.; Perinot, A.; Chen, Z.; Facchetti, A.; Caironi, M. 
Macroscopic and high-throughput printing of aligned nanostructured polymer semiconductors for $\mathrm{MHz}$ large-area electronics. Nat. Commun. 2015, 6, 8394.

(21) Diao, Y.; Zhou, Y.; Kurosawa, T.; Shaw, L.; Wang, C.; Park, S.; Guo, Y.; Reinspach, J. A.; Gu, K.; Gu, X.; Tee, B. C. K.; Pang, C.; Yan, H.; Zhao, D.; Toney, M. F.; Mannsfeld, S. C. B.; Bao, Z. Flowenhanced solution printing of all-polymer solar cells. Nat. Commun. 2015, 6, 7955.

(22) Kim, N. K.; Jang, S. Y.; Pace, G.; Caironi, M.; Park, W. T.; Khim, D.; Kim, J.; Kim, D. Y.; Noh, Y. Y. High-Performance Organic Field-Effect Transistors with Directionally Aligned Conjugated Polymer Film Deposited from Pre-Aggregated Solution. Chem. Mater. 2015, 27, 8345-8353.

(23) Lei, T.; Wang, J. Y.; Pei, J. Design, synthesis, and structureproperty relationships of isoindigo-based conjugated polymers. Acc. Chem. Res. 2014, 47, 1117-1126.

(24) Zhang, M.; Tsao, H. N.; Pisula, W.; Yang, C.; Mishra, A. K.; Müllen, K. Field-effect transistors based on a benzothiadiazolecyclopentadithiophene copolymer. J. Am. Chem. Soc. 2007, 129, 3472-3473.

(25) Yan, H.; Chen, Z.; Zheng, Y.; Newman, C.; Quinn, J. R.; Dötz, F.; Kastler, M.; Facchetti, A. A high-mobility electron-transporting polymer for printed transistors. Nature 2009, 457, 679-686.

(26) Ha, J. S.; Kim, K. H.; Choi, D. H. 2,5-Bis(2-octyldodecyl)pyrrolo[3,4-c]pyrrole-1,4-(2H,5H)-dione-based donor-acceptor alternating copolymer bearing $5,5^{\prime \prime}$-di(thiophen-2-yl)-2,2"'-biselenophene exhibiting $1.5 \mathrm{~cm} 2 . \mathrm{V}(--1) . \mathrm{s}(--1)$ hole mobility in thin-film transistors. J. Am. Chem. Soc. 2011, 133, 10364-10367.

(27) Guo, X.; Puniredd, S. R.; Baumgarten, M.; Pisula, W.; Müllen, $\mathrm{K}$. Rational design of benzotrithiophene-diketopyrrolopyrrole-containing donor-acceptor polymers for improved charge carrier transport. Adv. Mater. 2013, 25, 5467-5472.

(28) Wang, S.; Fabiano, S.; Himmelberger, S.; Puzinas, S.; Crispin, X.; Salleo, A.; Berggren, M. Experimental evidence that short-range intermolecular aggregation is sufficient for efficient charge transport in conjugated polymers. Proc. Natl. Acad. Sci. U. S. A. 2015, 112, 1059910604.

(29) Kang, I.; An, T. K.; Hong, J. A.; Yun, H. J.; Kim, R.; Chung, D. S.; Park, C. E.; Kim, Y. H.; Kwon, S. K. Effect of selenophene in a DPP copolymer incorporating a vinyl group for high-performance organic field-effect transistors. Adv. Mater. 2013, 25, 524-528.

(30) Chen, B.; Yang, Y.; Cheng, P.; Chen, X.; Zhan, X.; Qin, J. Designing a thiophene-fused DPP unit to build an A-D-A molecule for solution-processed solar cells. J. Mater. Chem. A 2015, 3, 68946900.

(31) Yao, J.; Yu, C.; Liu, Z.; Luo, H.; Yang, Y.; Zhang, G.; Zhang, D. Significant Improvement of Semiconducting Performance of the Diketopyrrolopyrrole-Quaterthiophene Conjugated Polymer through Side-Chain Engineering via Hydrogen-Bonding. J. Am. Chem. Soc. 2016, 138, 173-185.

(32) Ashraf, R. S.; Meager, I.; Nikolka, M.; Kirkus, M.; Planells, M.; Schroeder, B. C.; Holliday, S.; Hurhangee, M.; Nielsen, C. B.; Sirringhaus, H.; McCulloch, I. Chalcogenophene Comonomer Comparison in Small Band Gap Diketopyrrolopyrrole-Based Conjugated Polymers for High-Performing Field-Effect Transistors and Organic Solar Cells. J. Am. Chem. Soc. 2015, 137, 1314-1321.

(33) Yang, J.; Wang, H.; Chen, J.; Huang, J.; Jiang, Y.; Zhang, J.; Shi, L.; Sun, Y.; Wei, Z.; Yu, G.; Guo, Y.; Wang, S.; Liu, Y. BisDiketopyrrolopyrrole Moiety as a Promising Building Block to Enable Balanced Ambipolar Polymers for Flexible Transistors. Adv. Mater. 2017, 29, 1606162.

(34) Nielsen, C. B.; Turbiez, M.; McCulloch, I. Recent Advances in the Development of Semiconducting DPP-Containing Polymers for Transistor Applications. Adv. Mater. 2013, 25, 1859-1880.

(35) Fei, Z.; Chen, L.; Han, Y.; Gann, E.; Chesman, A. S. R.; McNeill, C. R.; Anthopoulos, T. D.; Heeney, M.; Pietrangelo, A. Alternating 5,5-Dimethylcyclopentadiene and Diketopyrrolopyrrole Copolymer Prepared at Room Temperature for High Performance
Organic Thin-Film Transistors. J. Am. Chem. Soc. 2017, 139, 80948097.

(36) Ni, Z.; Dong, H.; Wang, H.; Ding, S.; Zou, Y.; Zhao, Q.; Zhen, Y.; Liu, F.; Jiang, L.; Hu, W. Quinoline-Flanked Diketopyrrolopyrrole Copolymers Breaking through Electron Mobility over $6 \mathrm{~cm}(2) \mathrm{V}-1$ s $(-1)$ in Flexible Thin Film Devices. Adv. Mater. 2018, 30, 1704843.

(37) Cortizo-Lacalle, D.; Arumugam, S.; Elmasly, S. E. T.; Kanibolotsky, A. L.; Findlay, N. J.; Inigo, A. R.; Skabara, P. J. Incorporation of fused tetrathiafulvalene units in a DPP-terthiophene copolymer for air stable solution processable organic field effect transistors. J. Mater. Chem. 2012, 22, 11310-11315.

(38) Grzybowski, M.; Glodkowska-Mrowka, E.; Stoklosa, T.; Gryko, D. T. Bright, Color-Tunable Fluorescent Dyes Based on pi-Expanded Diketopyrrolopyrroles. Org. Lett. 2012, 14, 2670-2673.

(39) Shi, D.; Liu, Z.; Ma, J.; Zhao, Z.; Tan, L.; Lin, G.; Tian, J.; Zhang, X.; Zhang, G.; Zhang, D. Half-Fused DiketopyrrolopyrroleBased Conjugated Donor-Acceptor Polymer for Ambipolar FieldEffect Transistors. Adv. Funct. Mater. 2020, 30, 1910235.

(40) Trilling, F.; Sachnik, O.; Scherf, U. $\pi$-Expanded diketopyrrolopyrroles as acceptor building blocks for the formation of novel donor-acceptor copolymers. Polym. Chem. 2019, 10, 627-632.

(41) Zhuang, W.; Bolognesi, M.; Seri, M.; Henriksson, P.; Gedefaw, D.; Kroon, R.; Jarvid, M.; Lundin, A.; Wang, E.; Muccini, M.; Andersson, M. R. Influence of Incorporating Different Electron-Rich Thiophene-Based Units on the Photovoltaic Properties of IsoindigoBased Conjugated Polymers: An Experimental and DFT Study. Macromolecules 2013, 46, 8488-8499.

(42) Pavlishchuk, V. V.; Addison, A. W. Conversion constants for redox potentials measured versus different reference electrodes in acetonitrile solutions at $25^{\circ} \mathrm{C}$. Inorg. Chim. Acta 2000, 298, 97-102.

(43) Bard, A. J.; Faulkner, L. R., Electrochemical Methods: Fundamentals and Applications. 2nd Ed. ed.; John Wiley \& Sons: New York, 2001.

(44) Stephens, P. J.; Devlin, F. J.; Chabalowski, C. F.; Frisch, M. J. Ab-Initio Calculation of Vibrational Absorption and CircularDichroism Spectra Using Density-Functional Force-Fields. J. Phys. Chem. 1994, 98, 11623-11627.

(45) Grimme, S.; Ehrlich, S.; Goerigk, L. Effect of the damping function in dispersion corrected density functional theory. J. Comput. Chem. 2011, 32, 1456-1465.

(46) Weigend, F.; Ahlrichs, R. Balanced basis sets of split valence, triple zeta valence and quadruple zeta valence quality for $\mathrm{H}$ to $\mathrm{Rn}$ : Design and assessment of accuracy. Phys. Chem. Chem. Phys. 2005, 7, $3297-3305$.

(47) Hirshfeld, F. L. Bonded-Atom Fragments for Describing Molecular Charge-Densities. Theor. Chim. Acta 1977, 44, 129-138.

(48) Lu, T.; Chen, F. Multiwfn: A multifunctional wavefunction analyzer. J. Comput. Chem. 2012, 33, 580-592.

(49) Gao, W.; Zhang, M.; Liu, T.; Ming, R.; An, Q.; Wu, K.; Xie, D.; Luo, Z.; Zhong, C.; Liu, F.; Zhang, F.; Yan, H.; Yang, C. Asymmetrical Ladder-Type Donor-Induced Polar Small Molecule Acceptor to Promote Fill Factors Approaching 77\% for HighPerformance Nonfullerene Polymer Solar Cells. Adv. Mater. 2018, 30, 1800052.

(50) Rappoport, D.; Furche, F. Property-optimized gaussian basis sets for molecular response calculations. J. Chem. Phys. 2010, 133, 134105 .

(51) Murray, J. S.; Politzer, P. The electrostatic potential: an overview. WIRes-Comput. Mol. Sci. 2011, 1, 153-163.

(52) Lu, T.; Chen, Q. van der Waals potential: an important complement to molecular electrostatic potential in studying intermolecular interactions. J. Mol. Model. 2020, 26, 315.

(53) Yanai, T.; Tew, D. P.; Handy, N. C. A new hybrid exchangecorrelation functional using the Coulomb-attenuating method (CAMB3LYP). Chem. Phys. Lett. 2004, 393, 51-57.

(54) Liu, Z.; Lu, T.; Chen, Q. An sp-hybridized all-carboatomic ring, cyclo[18]carbon: Electronic structure, electronic spectrum, and optical nonlinearity. Carbon 2020, 165, 461-467. 
(55) Baeg, K. J.; Khim, D.; Jung, S. W.; Kang, M.; You, I. K.; Kim, D. Y.; Facchetti, A.; Noh, Y. Y. Remarkable enhancement of hole transport in top-gated $\mathrm{N}$-type polymer field-effect transistors by a high-k dielectric for ambipolar electronic circuits. Adv. Mater. 2012, 24, 5433-5439.

(56) Fabiano, S.; Usta, H.; Forchheimer, R.; Crispin, X.; Facchetti, A.; Berggren, M. Selective remanent ambipolar charge transport in polymeric field-effect transistors for high-performance logic circuits fabricated in ambient. Adv. Mater. 2014, 26, 7438-7443.

(57) James, D. I.; Wang, S.; Ma, W.; Hedström, S.; Meng, X.; Persson, P.; Fabiano, S.; Crispin, X.; Andersson, M. R.; Berggren, M.; Wang, E. High-Performance Hole Transport and Quasi-Balanced Ambipolar OFETs Based on D-A-A Thieno-benzo-isoindigo Polymers. Adv. Electron. Mater. 2016, 2, 1500313. 